Return to the Manage Active Submissions page at http://spie.org/app/submissions/tasks.aspx and approve or disapprove this submission. Your manuscript will not be published without this approval. Please contact author_help@spie.org with any questions or concerns.

\title{
MR images from fewer data
}

\author{
Bahareh Vafadar and Philip J. Bones \\ Computational Imaging Group, Department of Electrical and Computer Engineering, University of Canterbury, \\ Christchurch, New Zealand
}

\begin{abstract}
There is a strong motivation to reduce the amount of acquired data necessary to reconstruct clinically useful MR images, since less data means faster acquisition sequences, less time for the patient to remain motionless in the scanner and better time resolution for observing temporal changes within the body. We recently introduced an improvement in image quality for reconstructing parallel MR images by incorporating a data ordering step with compressed sensing (CS) in an algorithm named 'PECS'. ${ }^{1}$ That method requires a prior estimate of the image to be available. We are extending the algorithm to explore ways of utilising the data ordering step without requiring a prior estimate. The method presented here first reconstructs an initial image $\mathbf{x}_{1}$ by compressed sensing (with sparsity enhanced by SVD), then derives a data ordering from $\mathbf{x}_{1}, R_{1}^{\prime}$, which ranks the voxels of $\mathbf{x}_{1}$ according to their value. A second reconstruction is then performed which incorporates minimisation of the first norm of the estimate after ordering by $R_{1}^{\prime}$, resulting in a new reconstruction $\mathbf{x}_{2}$. Preliminary results are encouraging.
\end{abstract}

Keywords: Parallel Magnetic Resonance Imaging (pMRI), Compressed Sensing (CS)

\section{INTRODUCTION}

In recent years compressed sensing (CS), also known as compressive sampling or sparse recovery, has attracted considerable attention in different areas of information theory, digital signal processing and imaging. The traditional Shannon-Nyquist sampling theorem specifies that a band limited analog signal can be perfectly reconstructed from captured samples if the sampling rate is at least twice the signal bandwidth. In applications like remote surveillance, spectroscopy, medical imaging and genomic data analysis, data acquisition at a high sampling rate is difficult to implement or sometimes physically impossible. To address this challenge, Candes and Romberg ${ }^{2}$ showed that it is practical to recover certain types of signals accurately from a small number of linear, random samples or measurements. Their results predict that it is possible to sense sparse signals by acquiring far fewer measurements than the Shannon-Nyquist limit using highly nonlinear methods. CS relies on the fundamental fact that many types of images and signals can be represented by using only a few non-zero coefficients in a suitable basis. These types of signals are called very sparse or highly compressible and lossy compression techniques like JPEG and MP3 exploit the property. A compression is obtained by storing only the largest basis coefficients. When full information of the signal is available, a reasonable strategy for reconstructing the signal is to set the non-stored coefficients to zero. However, when the signal first has to be acquired by a difficult measurement (sensing) procedure, this method is a waste of resources, since after a large effort is made to obtain full information on the signal, most of the information is thrown away at the compression stage.

Of course measuring directly the large coefficients as suggested above without knowing a priori their locations is not possible. However it has been shown that the precise number of required measurements for an accurate recovery of compressed version of the original signal is comparable to the compressed size of the signal. ${ }^{2}$ Two principles make sensing a sparse object by taking as few measurements possible: sparsity, which pertains to the signals of interest, and incoherence, which pertains to the sensing modality. As stated above, sparsity expresses that many natural signals are sparse or compressible in the sense that they have concise representations when expressed in the proper basis. Incoherence reveals the idea that signals having a sparse representation in any defined domain must be spread out in the domain in which they are acquired. ${ }^{3,4}$ An incoherent measurement system is shown to inherently satisfy the restricted isometry property (RIP). ${ }^{5}$ Since the signal is greatly undersampled, the linear system describing the measurements is underdetermined and therefore has infinitely many solutions. The key idea in CS is that the sparsity helps in isolating the original vector by using efficient algorithms. The reconstruction algorithm searches for the sparsest vector that is consistent with the linear measurements. This idea leads to the combinatorial $l_{0}$-norm or $l_{p}$-norm problems. ${ }^{4}$

Magnetic resonance imaging (MRI) is a non-invasive and highly flexible medical imaging modality with three dimensional capabilities. In conventional Cartesian Fourier transform-based imaging, samples are acquired in spatial Fourier

Further author information: E-mail: phil.bones@canterbury.ac.nz

8500 - 2 V. 2 (p.1 of 12) / Color: No / Format: Letter / Date: 8/10/2012 3:19:11 PM

SPIE USE: DB Check, Prod Check, Notes: 
Return to the Manage Active Submissions page at http://spie.org/app/submissions/tasks.aspx and approve or disapprove this submission. Your manuscript will not be published without this approval. Please contact author_help@spie.org with any questions or concerns.

space ("k-space") along readout lines uniformly spaced in the phase-encode directions. Then the image is reconstructed using an inverse discrete Fourier transform. The time required to sample enough points of k-space for the desired field of view (FOV), resolution, and signal-to-noise ratio (SNR) is limited by the inherent spin relaxation properties of the material and by the technical and physiologic problems associated with rapidly switched magnetic field gradients. This time-consuming nature of image encoding has inspired novel data acquisition and image reconstruction techniques. For example parallel imaging uses multiple receiver coils to reduce the scan time. In parallel MRI (pMRI) an array of coils is used to collect undersampled k-space data simultaneously without the need of further increased gradient performance. The different spatial sensitivity profiles of receiver coils are used for spatial encoding to produce high-quality images without aliasing. ${ }^{6}$ Despite the many advances in pMRI, there is always a need for further increases in the speed of image acquisition. CS has generated significant interest in MRI due to its potential for increasing data acquisition speed. Since sparse representation is one of the crucial requirements in successful recovery in CS MRI, different orthogonal transformations to sparsify the images were proposed and investigated. In early CS MRI work, classical analytical transforms such as total-variation, ${ }^{3}$ identity transform for angiograms, ${ }^{3}$ nonsubsampled contourlet transform, ${ }^{7}$ combined transforms ${ }^{8}$ and dual-tree complex wavelets transform ${ }^{9}$ were employed. These constraints impose uniqueness to the solution of ill-posed problems. More recently, to achieve high quality reconstruction results with high reduction factors in data sampling, adaptive transforms (or dictionaries) were focused rather than blindly and uniformly enforcing traditional methods. Data-driven adaptive redundant dictionaries, ${ }^{10}$ reference-guided sparsifying transform ${ }^{11}$ and K-SVD dictionary learning ${ }^{12}$ are some examples in this area.

In a previous paper ${ }^{1}$ we demonstrated an improvement in image quality in reconstructing parallel MR images by incorporating a data ordering step with compressed sensing in an algorithm named 'prior estimation-based compressed sensing' (PECS). That method requires a prior estimate of the image to be available. ${ }^{1}$ Extending the use of data sorting as first proposed by Adluru and DiBella, ${ }^{13}$ PECS allows prior knowledge of the underlying image to be intrinsically incorporated in the image recovery process. In Adluru and DiBella's proposal, the intensities of the signal estimate are reordered according to a preprocessing step when applying the constraints on the estimated solution within the iterative reconstruction. ${ }^{13}$ The ordering of the intensities was monotonic and thus minimized the finite differences norm if the correct image was estimated (the constraint Adluru and DiBella used in their CS work was the total-variation transform). An undersampled measured dataset was used for the estimated ordering. In PECS, the required sorting order was obtained from rearranging the elements in the underlying image based on the magnitude information gathered from a prior image estimate, so that the underlying image can be recovered in a new form that exhibits a higher level of sparsity. The prior image used for sorting order was estimated from the fully sampled central regions of k-space, i.e., a low resolution reconstruction. Comparing the results proved that the PECS reconstruction led to better image contrast and details. ${ }^{1}$

In the following we extend the algorithm by exploring ways of utilising the data ordering step without requiring a prior estimate. We first reconstruct an initial image $\mathbf{x}_{1}$ by CS, then derive a sorting order from $\mathbf{x}_{1}, R_{1}$, which ranks the voxels of $\mathbf{x}_{1}$ according to their value. A new reconstruction is performed which incorporates $R_{1}$ and results in a new reconstruction $\mathbf{x}_{2}$. Preliminary results are encouraging. This paper is organized as follows. First, we provide the necessary background on CS MRI and then in pMRI. In the remainder of the paper we describe the proposed algorithm and show results illustrating the performance of our algorithm. The final section provides discussion and conclusions.

\section{METHODS}

In principle, $\mathrm{MR}$ images can be reconstructed from a sequence of acquisitions using various k-space trajectories which cover a whole region of k-space. Conventionally the k-space sampling pattern needs to be designed to satisfy the ShannonNyquist sampling criterion, which in turn depends on the resolution and field of view (FOV). Having different sparsifying transforms for MR images and the nature of MR acquisition are two key properties enabling CS to suceed in MRI. Reconstruction from Cartesian acquisitions is as simple as applying the inverse Fast Fourier Transform (FFT) to k-space dataset. In this work we consider 2D Cartesian pMRI imaging.

\subsection{CS MRI}

The goal in CS MRI is to reconstruct the unknown image from limited k-space measurements. Consider a finite-length, discrete-time signal $\mathbf{f}$, which can be viewed as an $N \times 1$ vector in $\Re^{N}$ with elements $f[n], n=1,2 \ldots, N$. (We treat an image by vectorizing it into a one-dimensional vector). The imaging process is commonly modeled as

$$
\mathbf{y}=\mathbf{F}_{u} \mathbf{f},
$$

where $\mathbf{F}_{u} \in \mathcal{C}^{\mathcal{M} \times \mathcal{N}}$ is the undersampled Fourier encoding matrix relating the unknown image $\mathbf{f}$ to the limited $\mathrm{k}$-space measurements $\mathbf{y} \in \mathcal{C}^{\mathcal{M}}$. In general, one would not expect to be able to accurately recover $\mathbf{f}$ when $M<N$. However 
Return to the Manage Active Submissions page at http://spie.org/app/submissions/tasks.aspx and approve or disapprove this submission. Your manuscript will not be published without this approval. Please contact author_help@spie.org with any questions or concerns.

it is possible to accurately estimate $\mathbf{f}$ if it is ' $K$-sparse' by itself or in some other transformation domain; with a high probability CS can reconstruct $\mathbf{f}$ exactly from only $M$ measurements, where $M=O(K \log (N / K)){ }^{2,14}$

It has been justified that measurement matrices constructed by randomly drawing entries from a certain distribution are largely incoherent with any fixed basis and ensure an exact recovery. ${ }^{4,14}$ Gaussian and Bernoulli measurement matrices are two examples of random matrices which can recover all sparse signals with high probability. ${ }^{15}$ For an orthonormal basis matrix, the use of a random measurement matrix leads to a sensing matrix that obeys the uniform uncertainty principle (UUP) with overwhelming probability. ${ }^{5}$ Lustig exhibited a uniform random distribution of samples in spatial frequency does not take into account the energy distribution of MR images in the center of k-space. ${ }^{3}$ Consequently they proposed a random variable-density sampling with denser sampling near the center of k-space which characterized the incoherence properties of pure random sampling. The designed nonuniform random undersampling leads to incoherency of the aliasing artifacts caused by k-space undersampling and has been employed in many CS MRI reconstruction methods.

Assuming for a moment that $\mathbf{f}$ exhibits sparsity in itself, one of the theoretically simplest ways to recover such a vector from its measurements is $l_{0}$-minimization. Consider the $l_{0}$-norm that counts the number of non-zero entries in $\mathbf{f}$, Therefore a $K$-sparse vector has $l_{0}$-norm equal to $K$. The modified optimization for Eq. (1) is

$$
\min _{\mathbf{f} \in \Re^{N}}\|\mathbf{f}\|_{0} \quad \text { subject to } \quad \mathbf{y}=\mathbf{F}_{u} \mathbf{f} .
$$

In practice, the sparsity of $\mathbf{f}$ may only be evident after some transformation. Any signal in $\Re^{N}$ can be represented in terms of a basis of $N \times 1$ vectors $\psi_{i}, i=1,2, \ldots, N$. For simplicity, assume that the basis is orthonormal. Thus a signal f can be expressed as

$$
\mathbf{f}=\sum_{i=1}^{N} s_{i} \psi_{i} \quad \text { or } \quad \mathbf{f}=\mathbf{\Psi} \mathbf{s}
$$

where the vectors $\psi_{i}$ are columns of the $N \times N$ basis matrix, $\boldsymbol{\Psi}=\left[\psi_{1}\left|\psi_{2}\right| \ldots \mid \psi_{N}\right]$ and $\mathbf{s}$ is a $N \times 1$ column vector of weighting coefficients. Since $\boldsymbol{\Psi}$ is assumed to be orthogonal, $s_{i}=\psi_{i}^{T} \mathbf{f}\left({ }^{T}\right.$ denotes transposition). Thus $\mathbf{s}$ is the equivalent representation of the signal in the $\boldsymbol{\Psi}$ domain.

The signal $\mathbf{f}$ is $K$-sparse if to a close approximation it is a linear combination of only $K$ basis vectors; that is, only $K$ of the coefficients $s_{i}$ in Eq. (3) are nonzero and $(N-K)$ are zero. The case of interest is when $K<<N$. The signal $\mathbf{f}$ is compressible if the representation has just a few large coefficients and many small coefficients.

By substituting $\boldsymbol{\Psi}$ from Eq. (3), y in Eq. (2) can be written as synthesis-based sparse reconstruction

$$
\min _{\mathbf{s} \in \Re^{N}}\|\mathbf{s}\|_{0} \quad \text { subject to } \quad \mathbf{y}=\mathbf{F}_{u} \mathbf{\Psi} \mathbf{s}
$$

Typical formulations of the CS reconstruction problem with the analysis-based and synthesis-based regularization are in the following Lagrangian setup:

$$
\hat{\mathbf{f}}=\underset{\mathbf{f}}{\arg \min }\left\|\mathbf{y}-\mathbf{F}_{u} \mathbf{f}\right\|_{2}^{2}+\lambda \Gamma(\mathbf{\Phi f}), \quad \hat{\mathbf{s}}=\underset{\mathbf{s}}{\arg \min }\left\|\mathbf{y}-\mathbf{F}_{u} \mathbf{\Psi} \mathbf{s}\right\|_{2}^{2}+\lambda \Gamma(\mathbf{s}),
$$

where the function $\Gamma($.$) is a variant of the l_{p}$-norm with $0 \leq p \leq 1$ to enforce sparsity in both cases. ${ }^{3} \lambda$ is the regularization parameter which balances the trade off between the acquired data consistency and sparsity of the image representation. The key property in designing or learning the sparsifying transform $\boldsymbol{\Psi}$ is its sparsifying ability to capture the local (spatial) contextual information in an image and suppress incoherent noise, i.e., an appropriate transform should produce high-magnitude sparse coefficients such that by imposing $\Gamma(\Phi \mathbf{f})$, the $l_{p}$-norm significantly constrains the solution space of f. ${ }^{11}$ The analytical total-variation transform imposes sparseness on the whole image domain such that finite differences in horizontal and vertical directions in the image are sparse. Typically this transform is considered as an additional constraint with the other proposed transforms to smooth the difference between the recovered values of adjacent pixels. For instance, artifacts usually exist in CS reconstructions when the wavelet transform is used alone for the sparse representation. Thus total variation (TV) terms are frequently combined with the wavelet transform to suppress these artifacts and enforce spatial homogeneity. ${ }^{3}$

Two distinct major approaches to sparse recovery have been presented with different benefits and shortcomings. $l_{1}$ minimization methods such as Basis Pursuit provides strong guarantees of exact recovery and stability, but relies on Linear Programming and carries the burden of polynomial time and increased implementation complexity. ${ }^{16}$ The second approach uses greedy methods (matching pursuit). While there is no guarantee that matching pursuit computes sparse representations, it is easily implemented, converges quickly, and has good approximation properties. ${ }^{17}$ 
Return to the Manage Active Submissions page at http://spie.org/app/submissions/tasks.aspx and approve or disapprove this submission. Your manuscript will not be published without this approval. Please contact author_help@spie.org with any questions or concerns.

\subsection{Sparsifying Transforms and Dictionaries}

According to restricted isometry property (RIP) condition, ${ }^{2}$ for a given amount of data, the sparser a signal is, the better the reconstruction that can be expected. Therefore sparse approximation is at the heart of many efficient compression and compressed sensing algorithms. However, fixed common orthogonal sparsifying transforms are not flexible enough to capture the complex characteristics of different signals of interest in particular applications. An extensive body of work investigated different sparsifying transforms that exploit the image characteristics for accurate reconstruction. ${ }^{\text {, }}{ }^{18-21}$ For example the wavelet transform fails to compress regular edges efficiently. $\operatorname{In}^{22}$ this problem was addressed by use of the curvelets transform. Recently, adaptive transforms (or dictionaries) have become increasingly popular as they can more accurately capture characteristics of the images during reconstruction. Instead of regularizing the CS inverse problem with a fixed basis, Peyré proposed a framework which uses sparsity in a a tree-structured dictionary of orthogonal bases which are adapted to a given image. ${ }^{19}$ Most of the work in dictionary-based recovery involves learning a dictionary from a collection of pre-collected reference images. The trained dictionary is then employed as the sparse representation basis for the CS recovery procedure. Learning sparsifying transform from a reference image to capture the anatomical structure of unknown image to guide the reconstruction process was demonstrated by Babican et al. ${ }^{11}$

\subsection{CS in Parallel MRI}

In recent years, Parallel MRI (pMRI) has been used for accelerating the MRI scans. In pMRI, multiple receiver coils are employed in data acquisition and due to the distinct sensitivity of each individual receiver coil to the object magnetization, the different coils receive somewhat independent measurements from the imaged object. Thus it is possible to recover the image using the undersampled data sets simultaneously acquired by the coils. To achieve further acceleration, both pMRI and CS-MRI were employed in our work. In the direct application of CS recovery with pMRI to accommodate multiple receiver measurements, the distinctive coil sensitivity encoding in different receiver coils needs to be employed . The $\mathrm{SENSE}^{6}$-like reconstruction formulation in $\mathrm{pMRI}$ is presented as follows:

$$
\hat{\mathbf{f}}=\underset{\mathbf{f}}{\arg \min }\|\mathbf{y}-\mathbf{E f}\|_{2}^{2}, \quad \mathbf{E}=\left[\begin{array}{c}
\mathbf{F}_{u} \operatorname{diag}\left(\mathbf{c}_{1}\right) \\
\mathbf{F}_{u} \operatorname{diag}\left(\mathbf{c}_{2}\right) \\
\vdots \\
\mathbf{F}_{u} \operatorname{diag}\left(\mathbf{c}_{M}\right)
\end{array}\right], \quad \mathbf{y}=\left[\begin{array}{c}
\mathbf{y}_{1} \\
\mathbf{y}_{2} \\
\vdots \\
\mathbf{y}_{M}
\end{array}\right],
$$

where $\mathbf{y}$ is the vector formed from $\mathrm{k}$-space data acquired in all the $M$ channels, $\mathbf{f}$ is the unknown vector defining the desired full (FOV) image to be computed, $\mathbf{E}$ is the sensitivity encoding matrix, $\mathbf{c}_{n}$ denotes the coil sensitivity profile for the $n$th coil, and $\operatorname{diag}\left(\mathbf{c}_{n}\right)$ is a diagonal matrix with the elements of vector $\mathbf{c}_{n}$ on the main diagonal and zero elsewhere. The direct generalization of CS in parallel imaging ${ }^{23,24}$ formulates the reconstruction from multi-channels, as the same convex optimization problem as CS-MRI in Eq. 5, except that the encoding matrix is the Fourier transform of the channel-specific sensitivity modulation $\mathbf{E}$, thus

$$
\hat{\mathbf{f}}=\underset{\mathbf{f}}{\arg \min }\|\mathbf{y}-\mathbf{E f}\|_{2}^{2}+\lambda \Gamma(\mathbf{\Phi f})
$$

\subsection{PECS}

Our group proposed 'prior estimation-based compressed sensing' (PECS) in a previous paper. ${ }^{1}$ PECS is a promising technique that exploits prior knowledge of the underlying image in pMRI reconstruction. It has been proved in various studies that the success of CS recovery is fundamentally limited by the sparsity level of the underlying image. The more compressible the signal, the sparser its Fourier, DCT, Wavelet, etc. transform is and consequently if we decrease $K$, then we can reconstruct the original signal from fewer samples.

Adluru and DiBella proposed a preprocessing technique to improve the reconstruction of the signals that may not fit the TV constraint well. ${ }^{13}$ Their method used non-reordered reconstructions to obtain information about the signal to be reconstructed to determine the orderings of the pixel intensities. They also proposed that ordering estimation can be performed from the low-resolution images when a variable density undersampling scheme is used, and from non-reordered constrained reconstructions. The TV norm corresponding to the optimal regularization parameter with an appropriate ordering, is lower than the corresponding optimal regularization parameter without reordering. Clearly, by reducing sudden variations in the rapidly varying curves, reordering gives a better match to the TV constraint.

Interestingly, reordering of data has recently found application in a very different application of compressed sensing. To enhance CS through improving signal compressibility a method by finding a sub-optimal permutation of wireless sensor networks was proposed. ${ }^{25}$ It was stated that their sub-optimal reordering stably maintains a more compressible view of 
Return to the Manage Active Submissions page at http://spie.org/app/submissions/tasks.aspx and approve or disapprove this submission. Your manuscript will not be published without this approval. Please contact author_help@spie.org with any questions or concerns.

the signal. The presented method increased signal reconstruction accuracy at the same spatial sampling rate. The signal vector was rearranged so that its sparse representation in the transform domain (the Fourier transform was used) has much of the energy of the signal concentrated on the first few frequency coefficients.

A data ordering step is a crucial component of PECS. The basis of the PECS algorithm is illustrated in Figure 1. Consider a 2D signal, extracted from a brain cross-sectional slice as shown in Fig. 1(a). In Fig. 1(b) the pixels of the image have been reordered to yield a monotonically increasing variation starting from the lower right corner. We depict the ordering process as applying the operator $R$. The discrete cosine transformation (DCT) coefficients of the two images were calculated and their magnitudes are depicted in Fig. 1(c) and Fig. 1(d), respectively. Note that many fewer coefficients appear to have substantial magnitude in Fig. 1(d) than in Fig. 1(c). Subsequently the highest magnitude $5 \%$ of the coefficients were retained and the rest relaxed to zero. Finally images were reconstructed from the resulting compressed data. Fig. 1(e) shows the result corresponding to applying conventional compression to the original image in Fig. 1a. Fig. 1(f) shows the result obtained after reconstructing the compressed version of Fig. 1(d), followed by an inverse ordering process $\left(R^{-1}\right)$. It is clear that the results for the case incorporating the reordering step are superior. A significant loss of high frequency information was resulted from the first decompression outcome.

To compare the reconstructed images the normalized mean square error (NMSE) between the reference signal and reconstructed one was calculated by:

$$
\mathrm{NMSE}=\sqrt{\frac{\sum_{i=1}^{P}\left(\left|I_{i}^{\text {reference }}\right|-\left|I_{i}^{\text {reconstructed }}\right|\right)^{2}}{\sum_{i=1}^{P}\left|I_{i}^{\text {reference }}\right|^{2}}},
$$

where $P$ denotes the number of elements within the reference signal. As reported in the captions for Fig. 1(e) and Fig. 1(f) the NMSE value for the reconstructed image utilizing the reordering step is significantly decreased.

In PECS, we applied the data sorting strategy to the CS recovery process. Hence, instead of the underlying signal $\mathbf{f}$ estimation, an attempt to recover the sorted signal $\mathbf{g}$ was made, where $\mathbf{f} \stackrel{\mathbf{R}}{\rightarrow} \mathbf{g}$. PECS is therefore formulated as follows:

$$
\hat{\mathbf{g}}=\underset{\mathbf{g}}{\arg \min }\left\|\mathbf{y}-\mathbf{E}_{R} \mathbf{g}\right\|_{2}^{2}+\lambda \Gamma(\mathbf{\Phi g}), \quad \hat{\mathbf{g}} \stackrel{R^{-1}}{\rightarrow} \hat{\mathbf{f}}
$$

where $\hat{\mathbf{f}}$ and $\hat{\mathbf{g}}$ are the estimates of the underlying signal and the sorted signal under sorting order $R$, respectively. In Eq. (9), $\mathbf{E}_{R}$ represents the sensitivity encoding matrix introduced in Eq. (6) with columns reordered according to $R$. The results presented showed that the data sorting allows the underlying signal to be recovered in an alternative form that possesses a higher level of sparsity, and hence leads to a better signal recovery. ${ }^{1}$

As exact knowledge of the underlying signal (image) is not available prior the reconstruction to gain the exact sorting order $(R)$, we used a signal prior estimate to give an approximate sorting order $R^{\prime}$. To perform PECS a low-resolution approximation of the image was achieved using only the central regions of k-space from multiple coil data sets. Then, the sorting order was obtained by sorting this low-resolution approximation into a 2D monotonical form, similar to Fig. 1. Although applying an approximate sorting order results in an imperfectly sorted signal, which is inherently less sparse than if $R$ was used, it appeared to modify the form of reconstruction artifacts. In general, applying a data sorting modifies the form of reconstruction artifacts in PECS from those in conventional CS. There are some structured artifacts in conventional CS recovery which are dependent on the specific transform used and the signal features. In PECS on the other hand, the structured artifacts present in the sorted signal/image domain and, after imposing the inverse ordering, are likely to be become unstructured in the final reconstruction. These high-frequency noise-like variations can be suppressed using a total variation constraint.

\subsection{Proposed Method: SPECS}

As a method of utilising the data ordering without requiring a prior estimation, we propose to reconstruct the initial image by applying singular value decomposition (SVD) to the CS algorithm. Classical approaches in CS consider non-adaptive, analytical transforms such as wavelets, total-variation, and contourlets. ${ }^{?, 3}$ A data-adaptive transform which captures important characteristics of the images of interest, can better reveal anatomical structures.

SVD has been used for texture characterization, image restoration and for facial recognition. ${ }^{26}$ SVD is an effective tool for minimizing data storage and data transfer. ${ }^{27}$ Since the approximation error for an image represented by SVD is simply the sum of all unused singular values, removing the lowest valued high-order values will not significantly reduce the approximation accuracy. Thus using SVD as the data-adaptive sparsity basis results in a sparse representation which can be employed in the CS algorithm.

The singular value decomposition of a matrix factors an $M \times N$ matrix A into the form 
Return to the Manage Active Submissions page at http://spie.org/app/submissions/tasks.aspx and approve or disapprove this submission. Your manuscript will not be published without this approval. Please contact author help@spie.org with any questions or concerns.

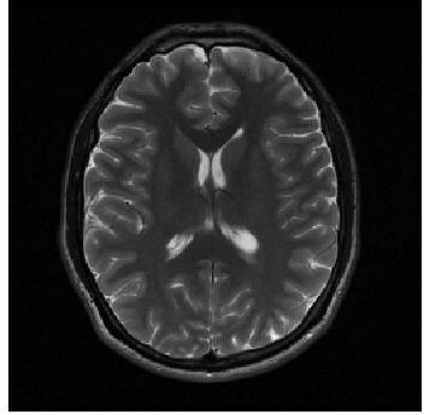

(a) Original 2D image

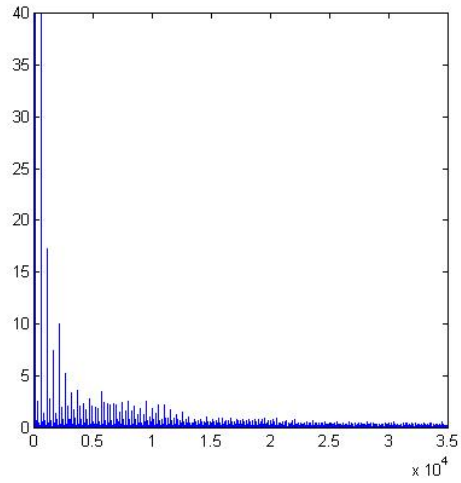

(c) DCT coefficients of the image

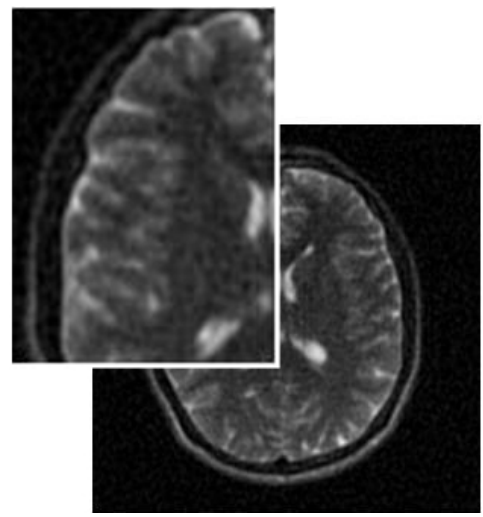

(e) Decompressed image

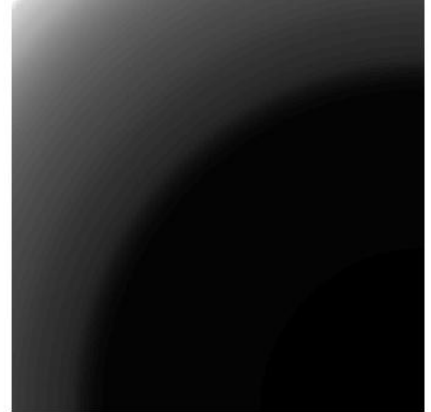

(b) Reordered image

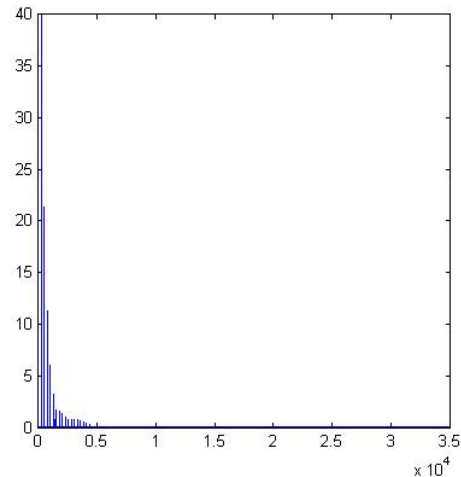

(d) DCT coefficients of the reordered image

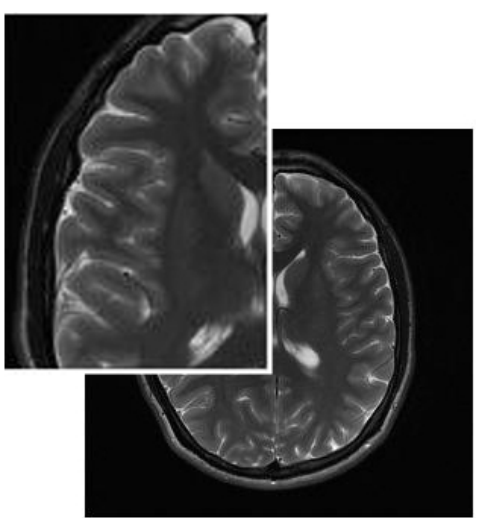

(f) Decompressed image after unsorting

Figure 1: (a) Illustrates the original image of an axial plane brain slice which reconstructed from full sampled $\mathrm{k}$-space and considered as the reference; (b) image (a) ordered into a a 2D monotonically decreasing variation $(R)$; (c) DCT coefficients of (a) for which the summation of the coefficients is 5947; (d) DCT coefficients of (b) for which summation of the coefficients is 472; (e) reconstructed image using only the $5 \%$ largest DCT coefficients from (c), NMSE $=0.1823$; (f) reconstructed image using only the $5 \%$ largest DCT coefficients from (d) and reordering $\left(R^{-1}\right), \mathrm{NMSE}=0.0018$. 


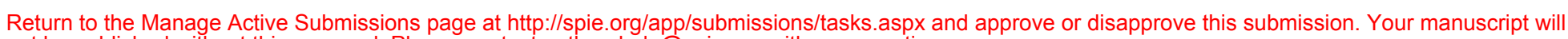
not be published without this approval. Please contact author_help@spie.org with any questions or concerns.

$$
\mathbf{A}=\mathbf{U} \boldsymbol{\Sigma} \mathbf{V}^{T}
$$

where $\mathbf{U}$ is an $M \times M$ orthonormal matrix; $\mathbf{V}$ an $N \times N$ orthonormal matrix, and $\boldsymbol{\Sigma}$ an $M \times N$ matrix containing the singular values of $\mathbf{A}$ along its main diagonal, arranged in descending order. Calculating the SVD consists of finding the eigenvalues and eigenvectors of $\mathbf{A} \mathbf{A}^{T}$ and $\mathbf{A}^{T} \mathbf{A}$. The eigenvectors of $\mathbf{A}^{T} \mathbf{A}$ make up the columns of $\mathbf{V}$ and the eigenvectors of $\mathbf{A} \mathbf{A}^{T}$ make up the columns of $\mathbf{U}$. The eigenvalues of both $\mathbf{A}^{T} \mathbf{A}$ and $\mathbf{A} \mathbf{A}^{T}$ are the squares of the singular values for A. Equation (10) can be expressed as

$$
\mathbf{A}=\sum_{i=1}^{p} \mathbf{u}_{i} \sigma_{i} \mathbf{v}_{i}^{T}, \quad \sigma_{1} \geq \sigma_{2} \geq \sigma_{3} \geq \cdots, \quad \sigma_{i}=0 \quad \text { for } \quad r<i \leq p
$$

where $r$ is the rank of matrix $\mathbf{A}, p$ is the smaller of the dimensions $M$ or $N$, and $\mathbf{u}_{i}, \mathbf{v}_{i}$ are the $i^{t h}$ column vectors of $\mathbf{U}$ $\mathbf{V}$ respectively, and $\sigma_{i}$ are the singular values. The matrix $\mathbf{A}$ can be approximated by matrix $\hat{\mathbf{A}}$ with rank $k$ by

$$
\hat{\mathbf{A}}=\sum_{i=1}^{k} \mathbf{u}_{i} \sigma_{i} \mathbf{v}_{i}^{T}
$$

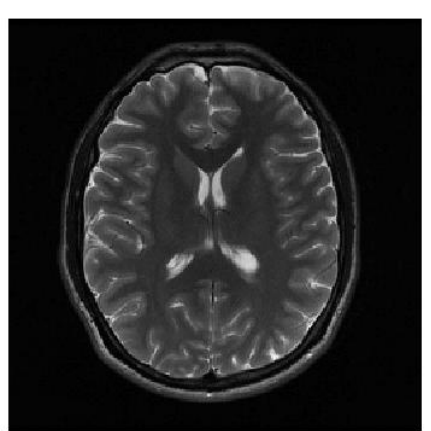

(a)

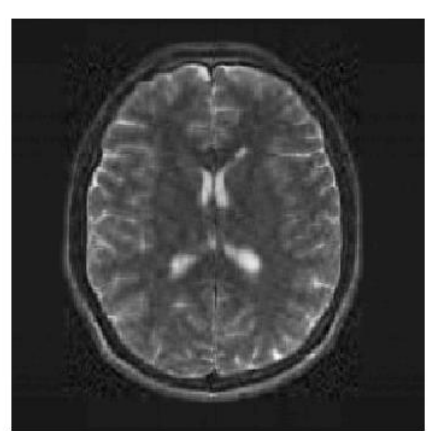

(b)

Figure 2: The results of an SVD-based image compression with different compression ratios: in (a) $50 \%$ and in (b) $10 \%$ of coefficients in $\boldsymbol{\Sigma}$ were used to accomplish compression. The original uncompressed image is shown in Fig. 1(a).

As the singular values are in descending order it can be seen that the error decreases towards zero in the 2-norm sense. In Fig. 2 the same image as in Fig. 1(a) was used to assess the compression obtainable via SVD. In Fig. 2(a) and Fig. 2(b) reconstructions are shown from $50 \%$ and $10 \%$ of the singular values, respectively.

Incorporating Equation (10) into the optimization expressed in Equation (7) allows reconstruction of an image with SVD as the sparsifying transform. Direct inverse Fourier transform from the undersampled k-space is used for the initial estimate (A) of the target $\mathbf{U}, \boldsymbol{\Sigma}$ and $\mathbf{V}$. The CS reconstruction with the SVD transformation recovered image is used to derive the data order approximation and that is incorporated into the second CS recovery framework. After applying the unsorting process to the second CS, a final reconstruction with high fidelity is obtained. We named this new method 'SPECS' since it represents an SVD-based modification of PECS. A summary of the SPECS algorithm is presented below: 
Please verify that (1) all pages are present, (2) all figures are correct, (3) all fonts and special characters are correct, and (4) all text and figures fit within the red margin lines shown on this review document. Complete formatting information is available at http://SPIE.org/manuscripts

Return to the Manage Active Submissions page at http://spie.org/app/submissions/tasks.aspx and approve or disapprove this submission. Your manuscript will not be published without this approval. Please contact author_help@spie.org with any questions or concerns.

\section{SPECS Algorithm}

Initialization:

Set $\mathbf{y}$ : under-sampled k-space measurements, $\mathbf{F}_{u}$ : under-sampled Fourier matrix

E: sensitivity encoding operator,

A: initial image reconstructed by inverse Fourier transformation of the under-sampled k-space data

SVD: sparsifying transform, image $\mathbf{A}$ to sparse representation $\boldsymbol{\Sigma}$

\section{CS algorithm:}

Solve The $l_{1}$-norm optimization defined in (7), with added total variation term, to reconstruct the image

SVD regularization parameter $\lambda_{1}=0.03$, TV regularization parameter $\lambda_{2}=0.01$

max number of iterations $=5$

Repeat the iterations in $\mathrm{NLCG}^{3}$ algorithm until the stopping criterion is met

Output: $\mathbf{x}_{1}$ reconstruction result which is used to derive sorting order $R_{1}^{\prime}$.

\section{Initialization:}

Set $\quad \mathbf{y}$ : under-sampled k-space measurements, $\mathbf{F}_{u}$ : under-sampled Fourier matrix

E: sensitivity encoding operator, DCT: sparsifying transform, $R_{1}^{\prime}$ : approximate sorting order

\section{PECS algorithm:}

Solve The $l_{1}$-norm optimization defined in (9), with added total variation term, to reconstruct the image

DCT regularization parameter $\lambda_{1}=0.02$, TV regularization parameter $\lambda_{2}=0.02$

max number of iterations $=30$

Repeat the iterations in NLCG algorithm until the stopping criterion is met

Output: reordered reconstruction

Invoke the unsorting process $\left(R^{\prime-1}\right)$ to form the final reconstructed image

Final output: $\mathrm{x}_{2}$

\section{EXPERIMENTS}

To evaluate the performance of the proposed SPECS method an in vivo experimental data set was acquired on a normal volunteer. The data used was obtained using a 1.5T GE scanner equipped with an eight-channel head coil. A T2weighted axial brain slice was obtained with the following parameters: FOV $=22 \mathrm{~cm}$, matrix size $=256 \times 256$, TR/TE $=2270 / 85 \mathrm{msec}$. The phase encoding and frequency encoding directions were set to be posterior/anterior and left/right, respectively. Consent from the volunteer was obtained prior to the scan. A full k-space data set was first obtained, then various forms of sampling patterns were applied to simulate the undersampling required. The sparsifying transforms used in the two phases of the algorithm were 2D SVD and 2D DCT. Lustigs nonlinear conjugate gradient descent toolbox ${ }^{3}$ was modified from its initial form and used in the iterative reconstruction. A sampling pattern suitable for CS is one of the key ingredients in a successful recovery. Therefore, the k-space data were randomly undersampled along the phase-encoding direction with denser sampling near the k-space center than near the higher frequency periphery.

Fig. 3 and Fig. 4 compare the reconstruction outcomes of CS, PECS, and the proposed SPECS reconstruction algorithms. In each subfigure within the figures the top left section of the image is expanded to allow closer inspection of the quality. The NMSE value (cf. Eq. (8)) is listed in the caption for each subfigure/reconstruction method.

In Fig. 3 the k-space dataset was undersampled at an acceleration factor of 2, in other words just $50 \%$ of the full set of k-space samples were retained. Fig. 3(a) depicts the reconstructed image from the k-space data, with all missing data points replaced by zero values and after a straightforward inverse DFT. A conventional CS reconstruction is shown in Fig. 3(b). It can be seen to generate a reconstruction with significant loss of image contrast and details, which is due to the lack of frequency components. The PECS reconstruction, incorporating a prior image approximation (a low resolution image) to derive a data ordering, is shown in Fig. 3(c). Finally, the same direct reconstruction as shown in Fig. 3(a) is decomposed by SVD for the first phase of the new SPECS algorithm. As seen Fig. 3(d), the final reconstruction by the SPECS method achieved a noticeably superior outcome with a significantly lower NMSE than the other methods, yet it did not require any prior estimate.

In Fig. 4 the comparison was performed applying a significantly higher value of acceleration (acceleration factor $=5$, or only $20 \%$ of the data acquired) on the same dataset. In general, it is seen that even after applying such a high degree of under-sampling, the proposed SPECS method achieves improved results compared with the other methods. 
Return to the Manage Active Submissions page at http://spie.org/app/submissions/tasks.aspx and approve or disapprove this submission. Your manuscript will not be published without this approval. Please contact author help@spie.org with any questions or concerns.

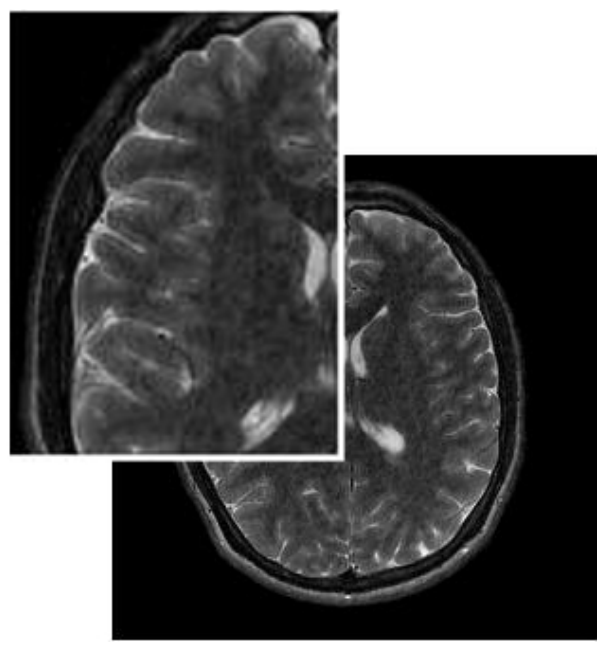

(a) Zero-filled reconstructed image

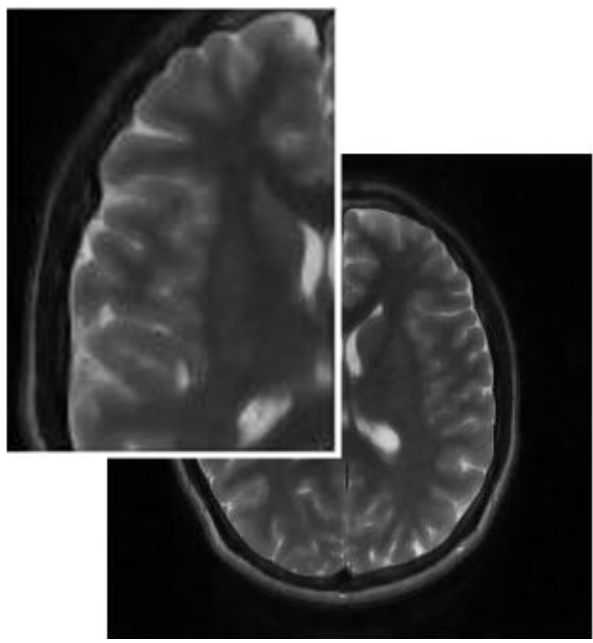

(c) PECS

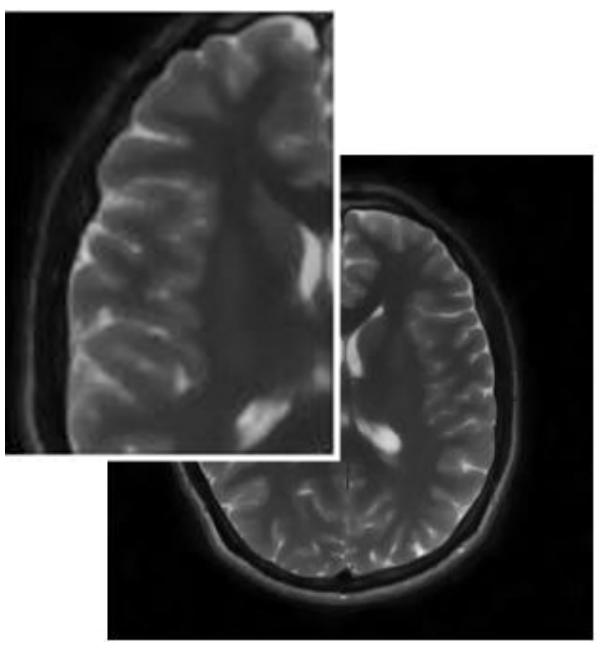

(b) CS

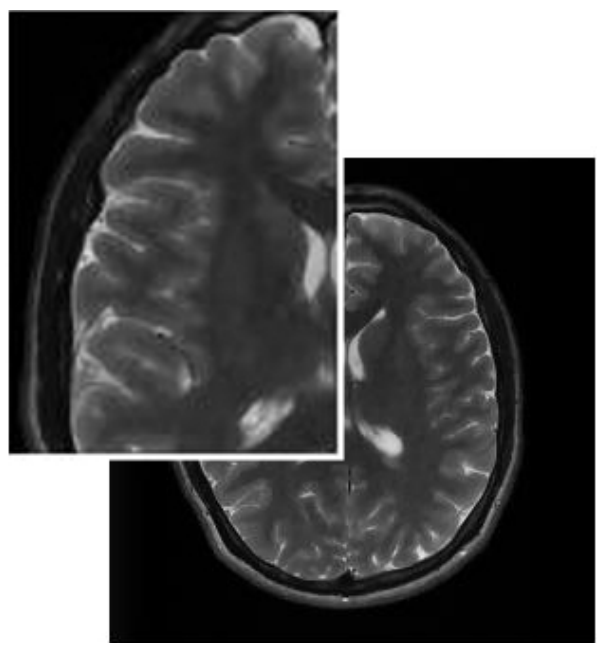

(d) SPECS

Figure 3: Reconstructed images from the undersampled k-space dataset for an acceleration factor $\mathbf{A F}=\mathbf{2}$ : (a) image reconstructed with zero-filled k-space, NMSE $=0.1840$; (b) reconstructed by the conventional CS method using the DCT as the sparsifying transform, NMSE $=0.1298$; (c) reconstructed image by the PECS method using a low resolution image to derive the sorting order, NMSE $=0.1320$; and (d) reconstructed image by the new SPECS method, NMSE $=0.0877$. 
Return to the Manage Active Submissions page at http://spie.org/app/submissions/tasks.aspx and approve or disapprove this submission. Your manuscript will not be published without this approval. Please contact author help@spie.org with any questions or concerns.

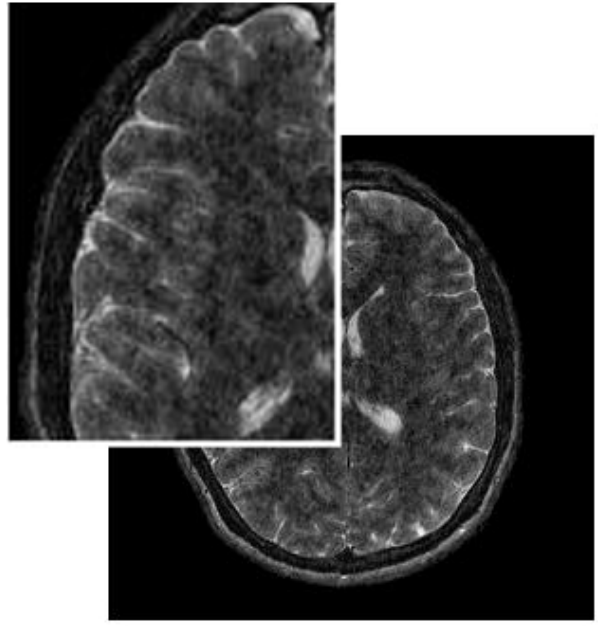

(a) Zero-filled reconstructed image

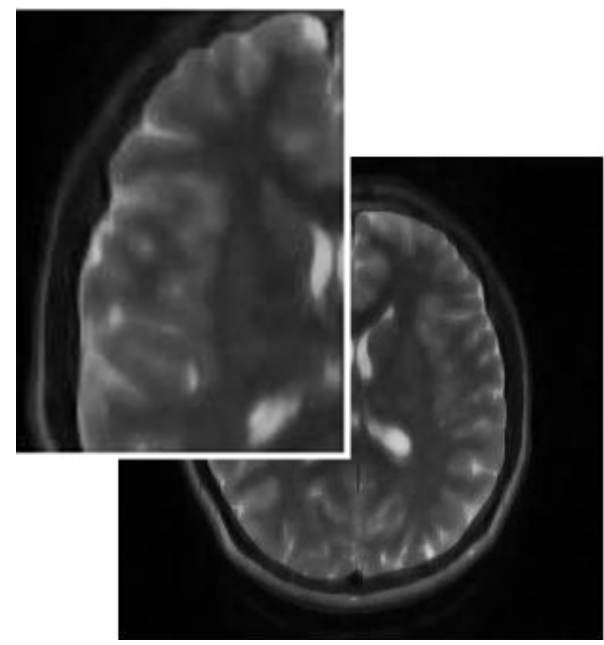

(c) PECS

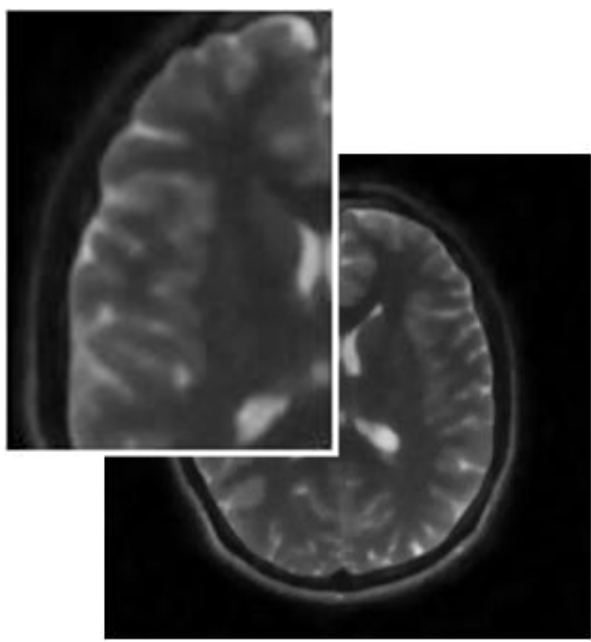

(b) CS

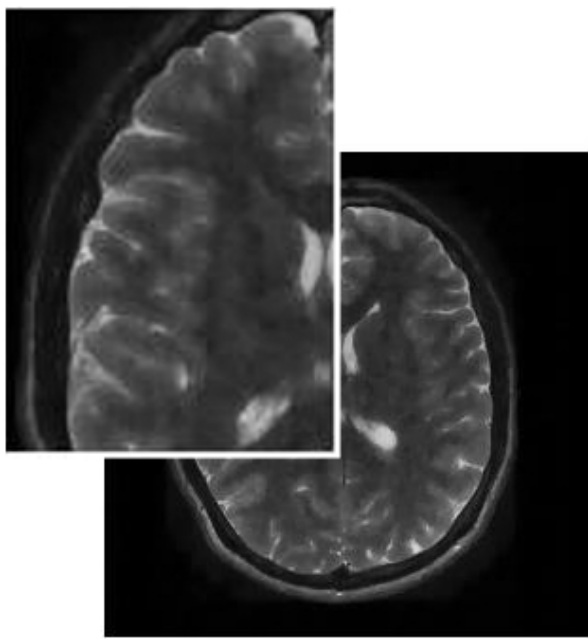

(d) SPECS

Figure 4: Reconstructed images from the undersampled k-space dataset for an acceleration factor $\mathbf{A F}=\mathbf{5}$ : (a) image reconstructed with zero-filled k-space; (b) reconstructed by the conventional CS method using the DCT as the sparsifying transform, NMSE $=0.1862$; (c) reconstructed image by the PECS method using a low resolution image to derive the sorting order, NMSE $=0.1751$; and (d) reconstructed image by the new SPECS method, $\mathrm{NMSE}=0.1352$. 
Return to the Manage Active Submissions page at http://spie.org/app/submissions/tasks.aspx and approve or disapprove this submission. Your manuscript will not be published without this approval. Please contact author_help@spie.org with any questions or concerns.

\section{DISCUSSION \& CONCLUSION}

Compressed sensing (CS) is a relatively new approach for the acquisition and recovery of incomplete information which exploits the inherent sparsity (explicit and implicit) of the underlying signal. The potential for incorporating image prior knowledge into CS recovery at high acceleration factors has been exploited here. Thus in PECS we had introduced a method for incorporating prior estimation of the underlying image in the reconstruction. Instead of recovering the transform of the image itself, the transform of a sorted image (based on the sorting required to place the pixels of a reference image in monotonic magnitude order) was recovered.

In the new method introduced here, SPECS, an alternative approach to capturing the underlying information needed for optimal reordering was employed: an adaptive, data-driven sparsifying transform was employed in CS algorithm to achieve an initial, relatively poor reconstruction, $\mathbf{x}_{1}$. SVD was chosen as the transform, since other studies suggested that it can reveal more underlying structure of the image than the other more commonly employed transforms. Using $\mathbf{x}_{1}$ to derive an approximate data ordering, $R_{1}^{\prime}$, makes the ordering process independent of prior image knowledge. The final reconstruction is performed as for PECS, exploiting the significant increase in sparsity the ordering process provides.

The preliminary results presented in Figs. 3 and 4 show It was presented that the recovered images after incorporating the obtained reordering in the second CS recovery, outperforms the PECS initial results. However SPECS requires no prior information about the image to be recovered, the only trade-off of this method is the slightly increased computational complexity compared to only using each method alone.

Despite the promising results achieved for compressed sensing in MRI, with pMRI there is difficulty in combining the requirements for a regular sampling pattern to exploit sensitivity encoding across the array of coil signals ${ }^{6}$ with the requirements for a random sampling pattern suited to $\mathrm{CS}^{3}{ }^{3}$ Recent findings ${ }^{28}$ have emphasized this difficulty. We argue that the data ordering step which has been incorporated into PECS and SPECS helps overcome this restriction. Consider the reconstruction with CS incorporating the DCT with that of SPECS: in the former case the compression combines a series of sinusoids giving rise to smoothly varying artifacts (loss of signal detail); in contrast, in SPECS the unsorting process causes the effective basis functions to becomes unstructured, resulting in the artifacts being distributed and effectively random in nature. We argue that they become less destructive and true image features are thereby more observable.

\section{ACKNOWLEDGEMENTS}

The authors acknowledge the support of the National Heart Foundation of New Zealand for this work.

\section{REFERENCES}

[1] Wu, B., Millane, R., Watts, R., and Bones, P., "Prior estimated-based compressed sensing in parallel MRI," Magn. Res. Med. 65(1), 83-95 (2011).

[2] Candés, E., Romberg, J., and Tao, T., "Stable signal recovery from incomplete and inaccurate measurements," Comm. Pure Appl. Math. 59(8), 1207-1223 (2006).

[3] Lustig, M., Donoho, D., and Pauly, J., "Sparse MRI: The application of compressed sensing for rapid MR imaging," Magn. Res. Med. 58(6), 1182-1195 (2007).

[4] Fornasier, M. and Rauhut, H., "Compressive sensing," in [Handbook of Mathematical Methods in Imaging], Scherzer, O., ed., 187-229, Springer (2010).

[5] Candés, E., "Compressive sampling," in [Proc. Int. Congress of Math. Madrid, Spain], 1433-1452 (2006).

[6] Pruessmann, K., Weiger, M., Scheidegger, M., and Boesiger, P., "SENSE: Sensitivity Encoding for fast MRI," Magn. Res. Med. 42(5), 952-962 (1999).

[7] Qu, X., Guo, D., Chen, Z., and Cai, C., "Compressed sensing MRI based on nonsubsampled contourlet transform," in [Proc. IEEE Int. Symp. IT Med. Educ. ITME08, Xiamen, China], 693-696 (2010).

[8] Qu, X., Cao, X., Guo, D., Hu, C., and Chen, Z., "Compressed sensing MRI with combined sparsifying transforms and smoothed $l_{0}$ norm minimization," in [Proc. IEEE Int. Conf. Acous. Speech Sig. Proc. (ICASSP), Dallas], 626-629 (2010).

[9] Kim, Y., Altbach, M., Trouard, T., and Bilgin, A., "Compressed sensing using dual-tree complex wavelet transform," in [Proc. 17th Scientific Meeting, Int. Society for Magn. Res. Med. ISMRM09, Honolulu], 17, 2814 (2009).

[10] Ni, Z., Zhang, M., Li, J., and Wang, Q., "Image compressed sensing based on data-driven adaptive redundant dictionaries," Progress In Electromagnetics Research 22, 73-89 (2012).

[11] Babacan, S., Peng, X., Wang, X., Do, M., and Liang, Z., "Reference-guided sparsifying transform design for compressive sensing MRI," in [Proc. IEEE Int. Conf. Eng. Med. Biol. Soc. EMBC], 5718-5721 (2011). 


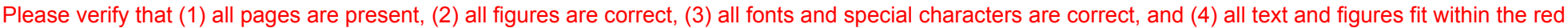
margin lines shown on this review document. Complete formatting information is available at http://SPIE.org/manuscripts

Return to the Manage Active Submissions page at http://spie.org/app/submissions/tasks.aspx and approve or disapprove this submission. Your manuscript will not be published without this approval. Please contact author_help@spie.org with any questions or concerns.

[12] Ravishankar, S. and Bresler, Y., "MR image reconstruction from highly undersampled k-space data by dictionary learning," IEEE Trans. Medical Imaging 30(5), 1028-1041 (2011).

[13] Adluru, G. and DiBella, E., "Reordering for improved constrained reconstruction from undersampled k-space data," Int. J. Biomedical Imaging 2008, 341684 (2008).

[14] Baraniuk, R., "Compressive sensing [lecture notes]," IEEE Signal Processing Magazine 24(4), 118-121 (2007).

[15] Tropp, J. and Gilbert, A., "Signal recovery from random measurements via orthogonal matching pursuit," IEEE Trans. Inf. Theory 53(12), 4655-4666 (2007).

[16] Needell, D., "Topics in compressed sensing," Ph.D. Dissertation, Math. , Univ. of California, Davis (2009).

[17] Gribonval, R. and Vandergheynst, P., "On the exponential convergence of matching pursuits in quasi-incoherent dictionaries," IEEE Trans. Inf. Theory 52(1), 255-261 (2006).

[18] Dong, Y. and Ji, J., "Compressive sensing MRI with laplacian sparsifying transform," in [Proc. IEEE Int. Conf. Biomedical Imaging: From Nano to Macro, Chicago], 81-84 (2011).

[19] Peyré, G., "Best basis compressed sensing," in [Proc. of Scale Space and Variational Methods in Computer Vision SSVM07], 80-91 (2007).

[20] Otazo, R. and Sodickson, D., "Adaptive compressed sensing MRI," in [Proc. of the 18th Sci. Mtg., Int. Soc. Magn. Res. Med., ISMRM10, Stockholm, Sweden], 4867 (2010).

[21] Baker, C., King, K., Liang, D., and Ying, L., "Translational-invariant dictionaries for compressed sensing in magnetic resonance imaging," in [Proc. 8th IEEE Int. Symp. on Biomedical Imaging: From Nano to Macro], 1602-1605 (2011).

[22] Candés, E. and Donoho, D., "New tight frames of curvelets and optimal representations of objects with piecewise C2 singularities," Comm. Pure Appl. Math. 57(2), 219-266 (2004).

[23] Wu, B., Watts, R., Millane, R., and Bones, P., "An improved approach in applying compressed sensing in parallel MR imaging," in [Proc. 17th Sci. Mtg., Int. Soc. Magn. Res. Med. ISMRM09, Honolulu], 4595 (2009).

[24] Liang, D., Liu, B., Wang, J., and Ying, L., "Accelerating SENSE using compressed sensing," Magn. Res. Med. 62(6), 1574-1584 (2009).

[25] Mahmudimanesh, M., Khelil, A., and Suri, N., "Reordering for better compressibility: Efficient spatial sampling in wireless sensor networks," in [Proc. IEEE Int. Conf. on Sensor Networks, Ubiquitous, and Trustworthy Computing SUTC, Newport Beach, California], 50-57 (2010).

[26] Muller, N., Magaia, L., and Herbst, B., "Singular value decomposition, eigenfaces, and 3d reconstructions," SIAM review 46(3), 518-545 (2004).

[27] Prasantha, H., Shashidhara, H., and Balasubramanya Murthy, K., "Image compression using SVD," in [Int. Conf. on Computational Intelligence and Multimedia Applications ICCIMA07], 3, 143-145 (2007).

[28] Nam, S., Akcakaya, M., Hu, P., Manning, W. J., V., T., and Nezafat, R., "Impact of coil sensitivity estimation on MRI reconstruction methods combining compressed sensing and parallel MRI," in [Proc. of the 18th Scientific Meeting, Int. Society for Magn. Res. Med. ISMRM10, Stockholm, Sweden], 518-545 (2010). 